\title{
THE EFFECT OF METAL POWDER ON THE OPTICAL PROPERTIES OF POLYVINYL ACETATE
}

\author{
Shurooq Sabbah ${ }^{1 *}$, Maher Hasan², Baiddaa Yehiaa ${ }^{3}$, Zenia Satar ${ }^{4}$ \\ ${ }^{* 1,2,3,4}$ Babylon University, College of Science, Department of Physics, Iraq
}

*Corresponding Author: -

\begin{abstract}
: -
In the present work, the effect of cupper particles onsomeoptical properties of Poly-vinyl acetate has been studied. For this purpose, many samples were prepared with different weight percentages of cupper particles. The samples were prepared by casting method with same thickness. The absorption has been recorded in the wavelength range (1901100)nm.The optical constants (absorption coefficient, extinction coefficient, energy gap)of the indirect allowed and forbidden transition, real and imaginary dielectric constants have been determined. The optical constants are increase with increasing of cupper particles concentration. The energy gap decreased with increasing the weight percentages of cupper particle.
\end{abstract}

Keywords: Polyvinyl acetate, Composite, Optical properties

\section{(a) (\$) (1)}




\section{INTRODUCTION}

Polymers are considered to be good hosting matrices for composite materials because they can easily be tailored to yield a variety of bulk physical properties. Moreover, organic polymers generally have long-term stability and good process ability. Polymers have relatively poor mechanical, thermal, and electrical properties as compared to metals and ceramics. Many types of polymers such as homopolymers, co-polymers, blended polymers and modified polymers are not sufficient enough to compensate various properties, which we have demanded. Alternative approaches to improve their properties are to reinforce polymers with inclusion of fiber, whisker, platelets, or particles. The choice of the polymers is usually guided mainly by their mechanical, thermal, electrical, optical and magnetic behaviors. However, other properties such as hydrophobic/hydrophilic balance, chemical stability, bio-compatibility, opto-electronic properties and chemical functionalities (i.e., solvation, wettability, templating effect, etc.) have to be considered in the choice of the polymers. The polymers in many cases can also allow easier shaping and better processing of the composite materials [1] If the band gaps of the materials were narrow, most of the incoming radiation would be absorbed by the electrons and be exited by the electrons from the valence band into the conduction band. If the materials, on the other hand, have wide energy band gap, the photon energy will be too weak and to cause any absorption and such materials would be seen as ineffective in converting the photon distribution to a narrow distribution. Only photons with energy greater than band gap of the materials will be absorbed. Photons of longer wavelength will pass through (i.e. be transmitted) having just sufficient energy to excite electrons [2].

The development of polymer systems with high ionic conductivity is one of the main objectives in polymer research. This is because of their potential applications as electrolytes in solid-state batteries, fuel cells, electrochemical display devices/smart windows, photo electrochemical cells etc., due to their high conductivity, high energy density, wide electrochemical stability and easy process ability. The main advantages of polymer electrolytes are their mechanical properties, ease of fabrication of thin films of desirable sizes and their ability to form proper electrode/electrolyte contact in electrochemical devices [3]. This paper deals with the effect of cupper on the optical properties of polyvinyl acetate.

\section{Experimental}

Polyvinyl acetate was dissolved in the distiller water by using magnetic stirrer. The different weights of cupper are (1.3, 1.4, 1.5and 1.6) wt.\% added to the polymer (PVAc). The solution was transferred to clean glass Petri dish of $(5.5 \mathrm{~cm})$ in diameter placed on plate form. The dried film was removed easily by using the tweezers clamp. The optical properties of composites were studied by using UV/1800/Shimadzu spectrophotometer.

\section{Results and discussion}

Absorptance $(A)$ can be defined as the ratio between absorbed light intensity $\left(I_{A}\right)$ by material and the incident intensity of light $\left(I_{o}\right)[4]$.

$$
A=\frac{I_{A}}{I_{0}}
$$

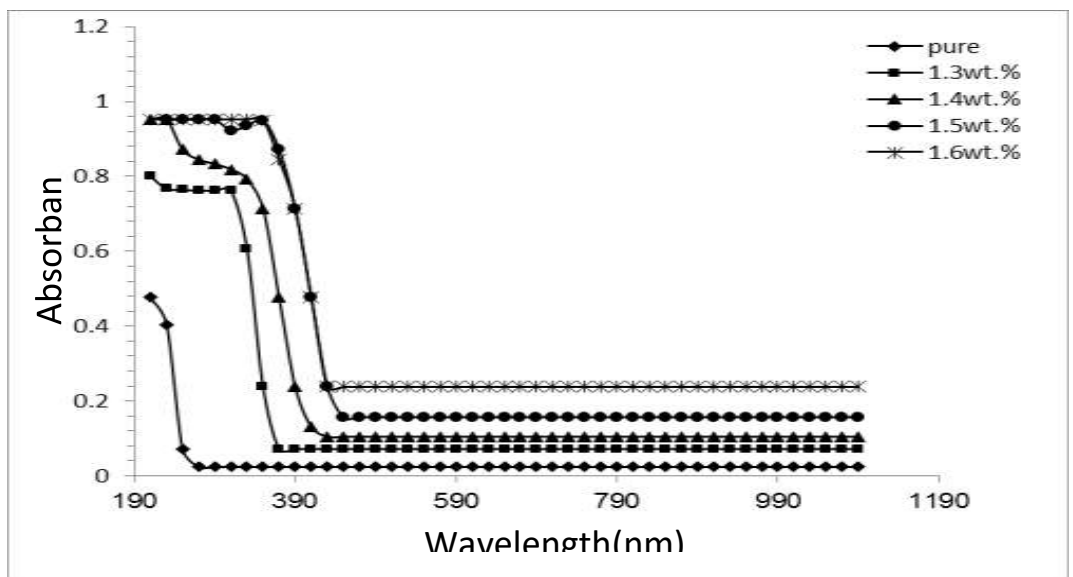

Figure (1): The absorbance as function of wavelength for (PVAc-Cu) composites.

Figure (1) shows the absorption spectrum of (PVAc-Cu) composites, as a function of the wavelength of the incident light. It can be noticed from the figure that the absorbance for all films have a high value at wavelength in the neighborhood of the fundamental absorption edge $(210 \mathrm{~nm})$, (390) for pure and weight of the cupper respectively, then the absorbance decreases with the increasing of wavelength. In general, the absorbance of films has low values in the visible and near infrared region. This behavior can be explained as follows: at high wavelength the incident photons don't have enough energy to interact with atoms, the photon will transmit. When the wavelength decreases, the interaction between incident photon and material will occur, and then the absorbance will increase [5]. In other words, absorb the incident light by the free electrons. Consequently, by the increase of the weight percentages of the cupper absorbance is increased. These results are similar to the results reached by the researchers [6,7]. 
3.2 The absoeption coefficient and energy gap of compsites.

Absorption coefficient is defined as a ratio decrement in flux of incident rays energy relative to the distance unit in the direction of incident wave diffusion. Absorption coefficient $(\alpha)$ depends on incident photon energy (hD) and on semiconductor characteristics ( $\mathrm{n}$ or $\mathrm{p}$ ) type, where electronic transitions type (n) or (p) and forbidden energy gap . Is give by following equation [8]:

$$
\alpha=2.303 \frac{A}{d}
$$

Where:A : Absorbance. $\boldsymbol{d}$ : The thickness of sample

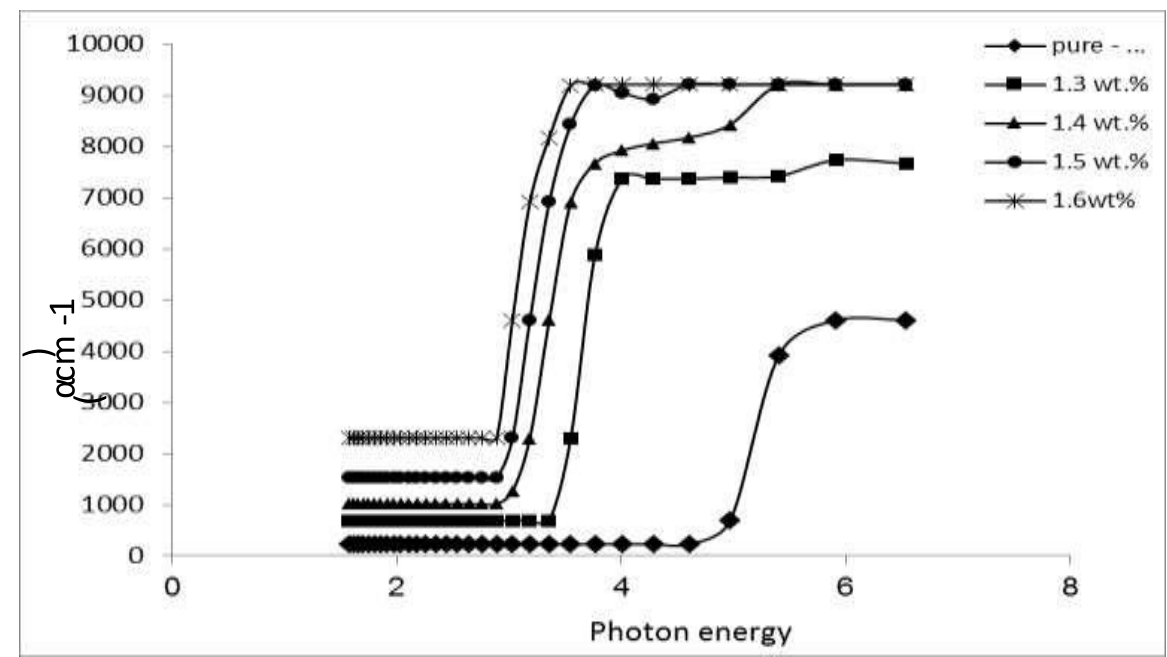

Figure (2): The absorption coefficient $\alpha(\mathrm{cm})^{-1}$ as a function of photon energy for (PVAcCu) composites.

Figures (2) shows the absorption coefficient $\alpha(\mathrm{cm})^{-1}$ as a function of photon energy for(PVAc-Cu)composites. It can be seen that the absorption coefficient is the smallest at low energy and high wavelength, this means that the possibility of electron transition is little because the energy of the incident photon is not sufficient to move the electron from the valence band to the conduction band $\left(\mathrm{h} v \square^{\mathrm{E}} \mathrm{g}\right)$. At high energies, absorption is bigger , this means that a great possibility for electron transitions consequently, the energy of incident photon is enough to move the electron from the valence band to the conduction band, the energy of the incident photon is greater than the forbidden energy gap[6].This shows that the absorption coefficient assists in figuring out the nature of electron transition, when the values of the absorption coefficient are high $\left(\alpha>10^{4}\right) \mathrm{cm}^{-1}$ at high energies it is expected that direct transition of electron occur ,the energy and moment are maintained by the electrons and photons. But when the values of the absorption coefficient are low $\left(\alpha<10^{4}\right) \mathrm{cm}^{-1}$ at low energies ,it is expected that indirect transition of electron occurs, and the electronic momentum is maintained with the assistance of the phonon [9], among other results is that the coefficient of absorption for the(PVAc- $\mathrm{Cu}$ ) composites is less than $10^{4} \mathrm{~cm}^{-1}$.

Figures (3) and (4) show the relation between absorption edge $(\alpha h v)^{1 / 2}$ and $(\alpha h v)^{1 / 3}$ for $(\mathrm{PVAcCu})$ composites as a function of photon energy respectively. Both the allowed and forbidden indirect transition band energy gap have been calculated by using following equation [10].

$$
\alpha h v=B\left(h v-E_{g}^{o p t .} \pm E_{p h}\right)^{r}
$$

Where: $\boldsymbol{h} \boldsymbol{v}$ is the energy of photon, $\boldsymbol{B}$ is proportionality constant, $\boldsymbol{E} \boldsymbol{g}$ is forbidden energy gap of the indirect transition. $\boldsymbol{E}_{\boldsymbol{p h}}$ : energy of phonon, is (-) when phonon absorption, and (+) when phonon emission.

$(\mathrm{r}=2)$ for the allowed indirect transition.

$(\mathrm{r}=3)$ for the forbidden indirect transition 


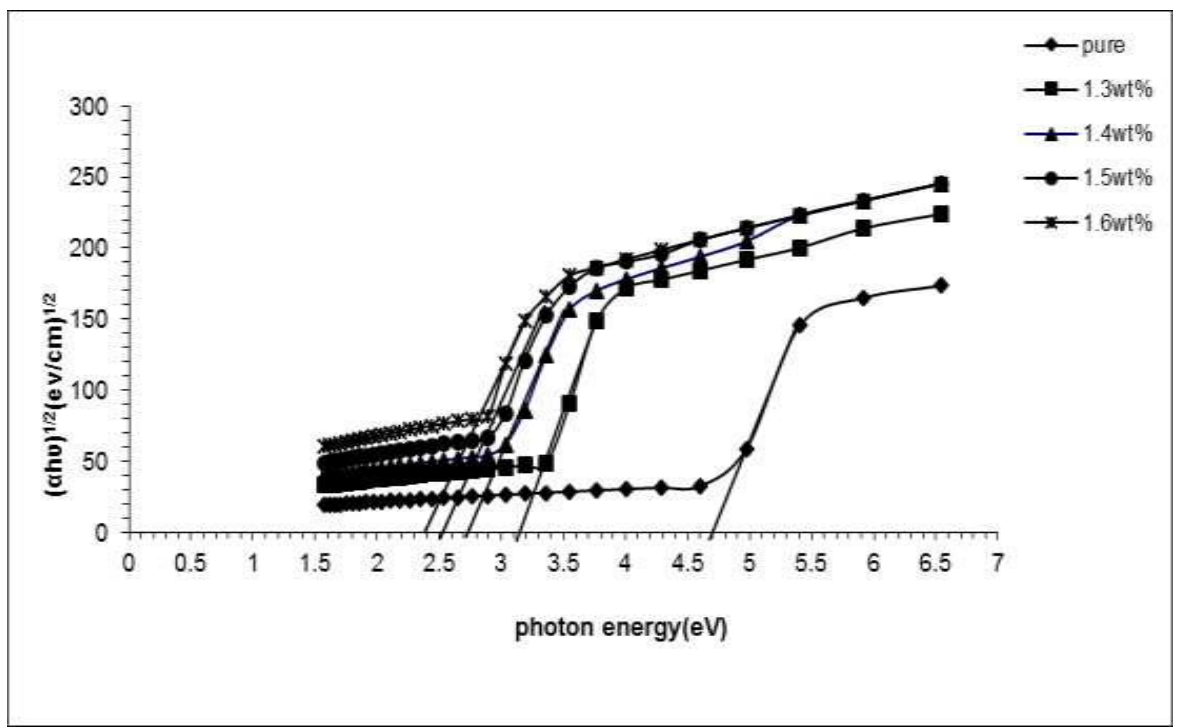

Figure (3): The energy gap for the allowed indirect transition $(\alpha h v)^{1 / 2}$ as a function of photon energy of (PVAc-Cu) composites

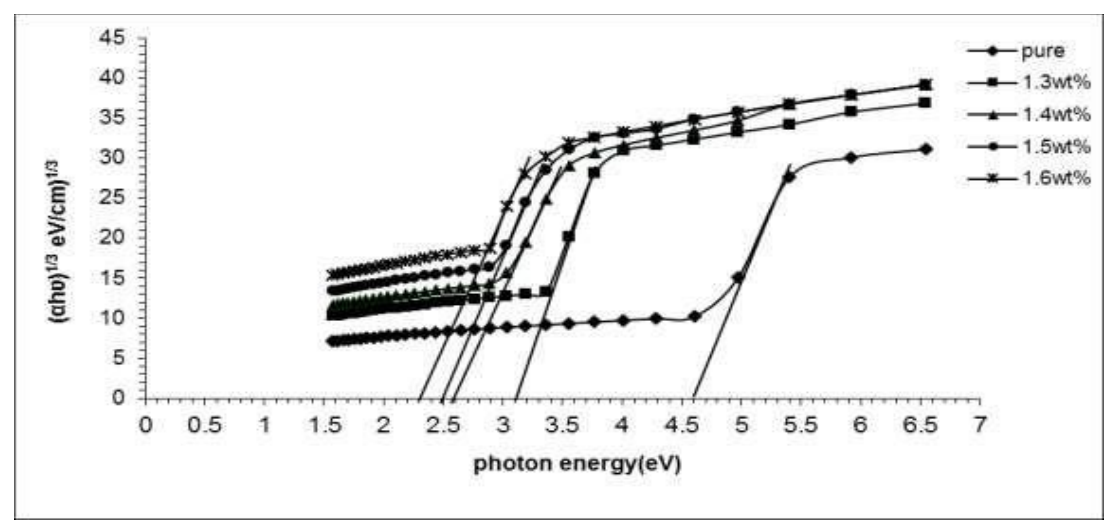

Figure (4): The energy gap for the forbidden indirect transition( $\alpha h v)^{1 / 3}$ as a function of photon energy of (PVAc-Cu) composites.

From the figures $(3,4)$ we can see the values of energy gap decrease with the increasing of the weight percentages of the cupper. The obtained values are shows in table. This attributed to the creation of site levels in the forbidden energy gap ,the transition in this case is conducted in two stages that involve the transition of electron from the valence band to the local levels to the conduction band as a result of increasing the weight percentages of the cupper. In other words the increase of the weight percentages of the cupper provides electronic paths in the polymer which facilitates the crossing of electron from the valance band to the conduction band,which explains the decrease of energy gap with the increase of the weight percentages of the cupper. These results are similar to the results reached by the researchers[6,7].

Table: The values of energy gap for the allowed and forbidden indirect transition for(PVAcCu)composites.

\begin{tabular}{|c|l|l|}
\hline \multirow{2}{*}{ The cupper wt.\% } & \multicolumn{2}{|c|}{$\mathrm{Eg}_{\mathrm{g}}(\mathrm{eV})$} \\
\cline { 2 - 3 } & allowed & forbidden \\
\hline 0 & 4.7 & 4.6 \\
\hline 1.3 & 3.2 & 3.1 \\
\hline 1.4 & 2.7 & 2.6 \\
\hline 1.5 & 2.6 & 2.5 \\
\hline 1.6 & 2.4 & 2.3 \\
\hline
\end{tabular}

\subsection{Extinction Coefficient $\left(K_{0}\right)$}

Extinction coefficient $\left(\mathrm{k}_{0}\right)$ is calculated by using following equation [11]:

$$
K_{0}=\frac{\alpha \lambda}{4 \pi}
$$

Where $\lambda:$ is the wavelength of incident photon rays 


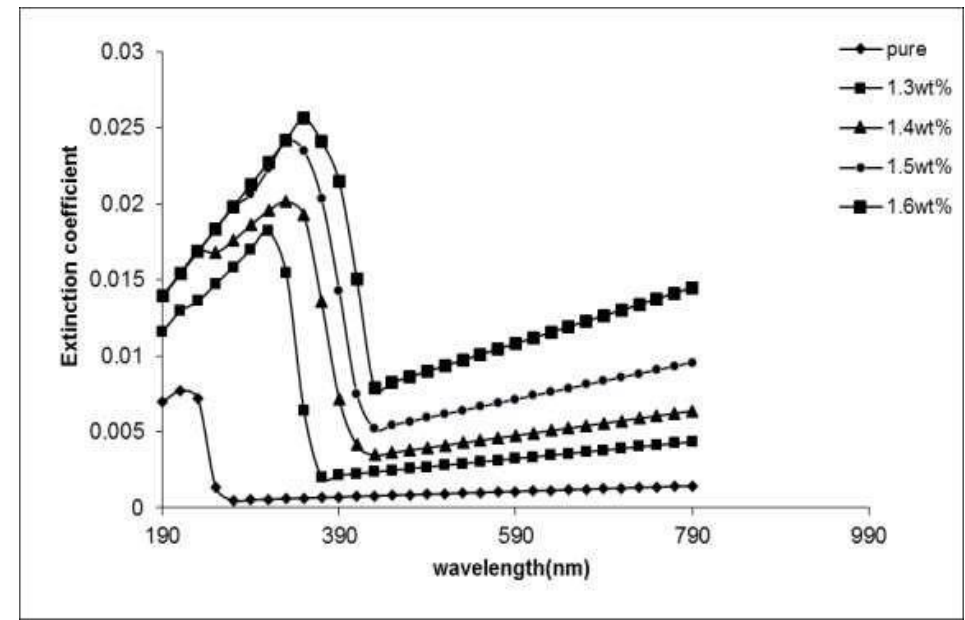

Figure (5): The Extinction coefficient as a function of wavelength for (PVAc-Cu) composites

Figure(5)shows the change of extinction coefficient for (PVAc-Cu) composites as a function of wavelength. From the figure we can see that $\left(\mathrm{k}_{0}\right)$ is low value at low concentration ,but it increases with the increasing of the concentration of the cupper( $\mathrm{Cu})$. This is attributed to increased absorption coefficient with the increase of weight percentages of $(\mathrm{Cu})$. Absorption coefficient has a direct relation with $\left(\mathrm{K}_{0}\right)$ as in the equation (4).

\subsection{Refractive Index(n)}

The refractive index can be defined as a ratio between velocity of light in vacuum (C), to its velocity inside the material. The value of refractive index (n) was calculated by using equation (5) depending on the reflectance and extinction coefficient $\left(\mathrm{K}_{\mathrm{o}}\right)$ as in the following equation [12].

$$
\mathrm{n}=\sqrt{\frac{4 \mathrm{R}-\mathrm{k}_{0}^{2}}{(\mathrm{R}-1)^{2}}}-\frac{(\mathrm{R}+1)}{(\mathrm{R}-1)}
$$

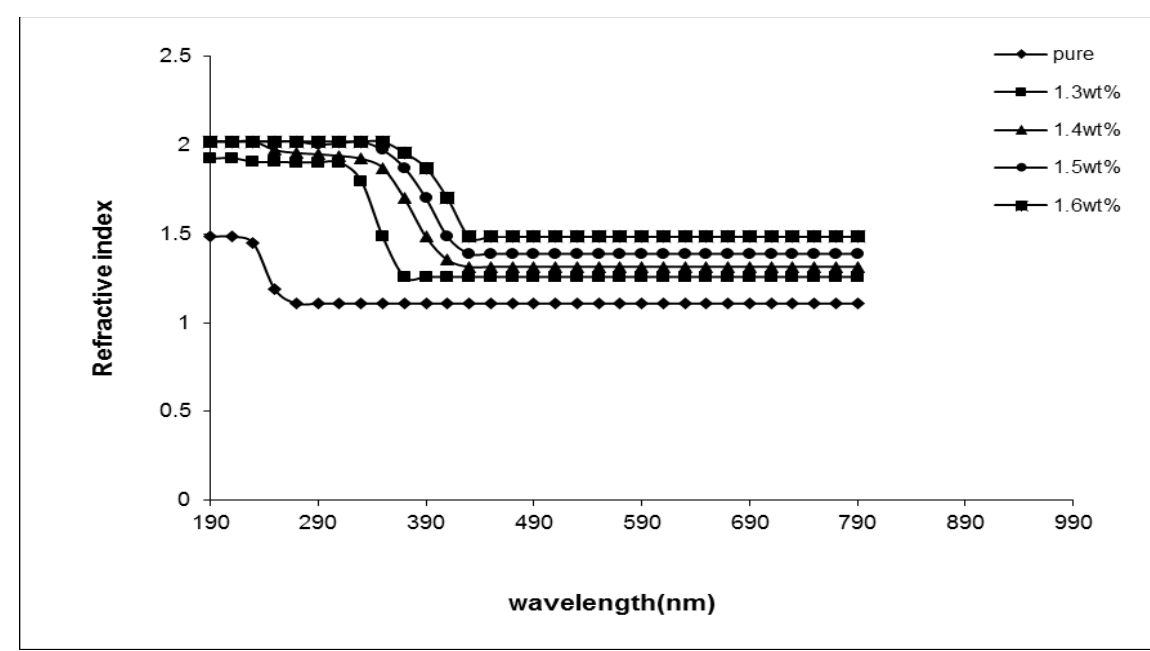

Figure (6): The refractive index(n)as a function of wavelength for (PVAc-Cu) composites

Figure (6) shows the change of refraction index for (PVAc-Cu) composites as a function of wavelength. From the figure we can see that the refractive index increases with increasing the weight percentages of the concentration of $(\mathrm{Cu})$ to the Polyvinyl acetate. The reason of this result is, the increase of the $(\mathrm{Cu})$ concentration leads to increase the density of the composites.

\subsection{Real ( $(\varepsilon 1)$ and Imaginary( $(2)$ part of Dielectricconstant}

Dielectric constant represents the ability of material to polarization, whose expression[7].The real and imaginary dielectric constant $\left(\varepsilon_{1}, \varepsilon_{2}\right)$ for (PVAc-Cu) composites have been calculated from following equations[8].

$$
\begin{aligned}
& \varepsilon_{1}=\left(n^{2}-K_{0}^{2}\right) \\
& \varepsilon_{2}=\left(2 n K_{0}\right)
\end{aligned}
$$




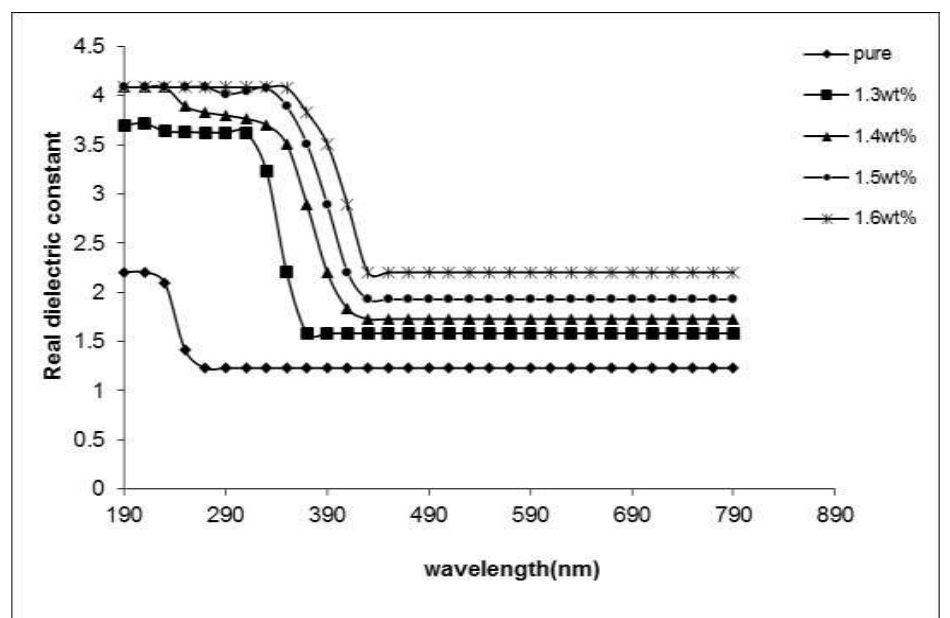

Figure (7):The real dielectric constant( $\left.\varepsilon_{1}\right)$ as a function of wavelength (PVAc-Cu) composites.

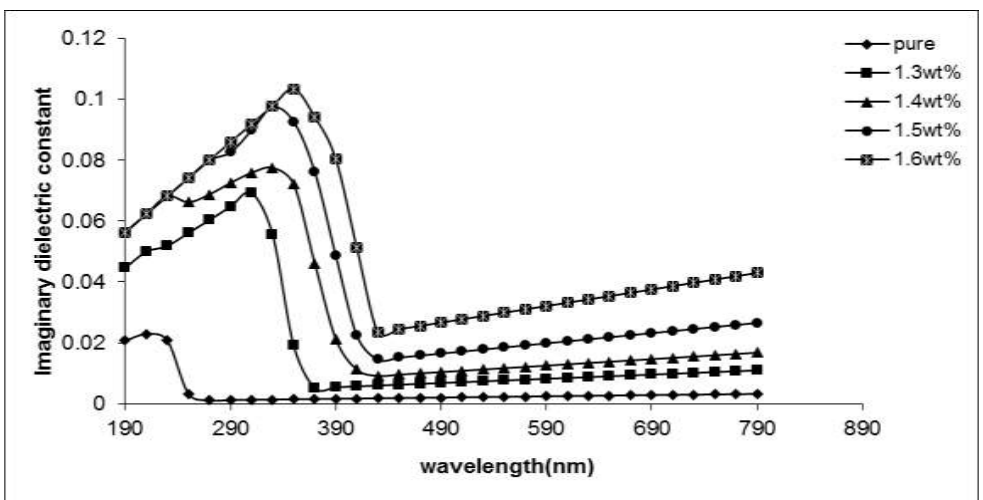

Figure (8): The The imaginary dielectric constant((2) as a function of wavelength (PVAc Cu) composites.

The figure (7) shows the change of $\left(\varepsilon_{1},\right)$ as a function of the wavelength. It can be seen that $\varepsilon_{1}$ considerably depends on $\left(\mathrm{n}^{2}\right)$ due to low value of $\left(\mathrm{k}_{0}^{2}\right)$ so, the real dielectric constant is increased with the increase of the concentration of $(\mathrm{Cu})$. Figure (8) show the change of $\left(\varepsilon_{2}\right)$ as a function of the wavelength. It can be seen that $\left(\varepsilon_{2}\right)$ is dependent on $\left(\mathrm{k}_{0}\right)$ values that change with the change of the absorption coefficient due to the relation between $(\alpha)$ and $(\mathrm{k} 0)$.

\section{Conclusions}

1. The absorbance and the absorption coefficient for (PVAc-Cu) composites increase with the increasing of the concentrations of the $(\mathrm{Cu})$ particles.

2. The absorption coefficient (PVAc-Cu) composites is less than $\left(10^{4}\right)(\mathrm{cm})^{-1}$ (happens indirect transition).

3. The energy gap of the transitions decreases with the increasing of the concentrations of the $(\mathrm{Cu})$ particles.

4. Extinction coefficient, refractive index and dielectric constant (real, imaginary) are increasing with the increasing of concentrations of the $(\mathrm{Cu})$ particles.

\section{References}

[1].[1] In-Yup Jeon and Jong-Beom Baek, "Nanocomposites Derived from Polymers and Inorganic Nanoparticles", J. of Materials, 3, 3654-3674.(2010).

[2].[2] E.I. Ugwu, "Optical Properties of Iron Halide ( $\mathrm{FeCl}$ ) Thin Film Deposited Using Solution Growth Technique $(S G T) "$, the Pacific Journal of Science and Technology, Vol. 7, No. 2. (2006).

[3].Lyly Nyl Ismail, Habibah Zulkefle, Sukreen Hana Herman, and Mohamad RusopMahmood, Influence of Doping Concentration on Dielectric, Optical, and Morphological Properties of PMMA Thin Films", J. of Advances in Materials Science and Engineering, Vol. 5, Article ID 605673, (2012).

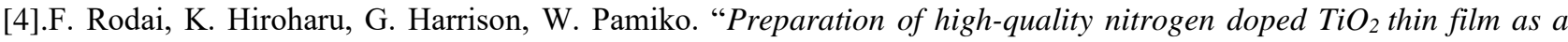
photocatalyst using a pulsed laser depositionmethod”. J. Thin Solid Films, 454, 162. (2004).

[5].Strectman B.G. and S.Banerjee, "Solid State electronic devices" ${ }^{\text {th }}$ ed., Engle Wood Cliffs, Nj" Prentice Hall (2000).

[6].M. Dahshan, "Introduction to Material Science and Engineering", $2^{\text {nd }}$ Ed. (2002).

[7].P.U.Asogwa"Band gap shift and optical characterization of PVA-Capped PbO thin films : Effect of thermal annealing"ChalcogenidLett.8,3 ,163-170, March (2011)

[8].J.I.Pankove, "Optical Process in Semiconductors", Dover Publishing, Inc., New York (1971).

[9].M. Crane, translation, Y. Hassan, "Solar Cells", Collage of Education, University of Mousl(1989).

[10]. A. N. Donald, "Semiconductor Physics and Devices", Irwin, USA, (1992).

[11]. T.Tsurumi, S. Nishizawa, N. Ohashi, T.1Ohagaki, “J.Appl.Phys.“, 38, 36-82(1999).

[12]. H.A.Macleod, "Thin Film Optical Filter", McGraw-Hill, New York. (2001). 